\title{
Induced acyclic subgraphs in random digraphs: Improved bounds
}

\author{
Kunal Dutta ${ }^{1}$ and C. R. Subramanian ${ }^{1}$ \\ ${ }^{1}$ The Institute of Mathematical Sciences, Taramani, Chennai - 600113, India. \\ email: $\{k d u t t a, c r s\} @ i m s c . r e s . i n$
}

Given a simple directed graph $D=(V, A)$, let the size of the largest induced directed acyclic graph (dag) be denoted by $\operatorname{mas}(D)$. Let $D \in \mathcal{D}(n, p)$ be a random instance, obtained by choosing each of the $\left(\begin{array}{l}n \\ 2\end{array}\right)$ possible undirected edges independently with probability $2 p$ and then orienting each chosen edge independently in one of two possible directions with probabibility $1 / 2$. We obtain improved bounds on the range of concentration, upper and lower bounds of $\operatorname{mas}(D)$. Our main result is that

$$
\operatorname{mas}(D) \geq\left\lfloor 2 \log _{q} n p-X\right\rfloor
$$

where $q=(1-p)^{-1}, X=W$ if $p \geq n^{-1 / 3+\epsilon}\left(\epsilon>0\right.$ is any constant), $X=W /(\ln q)$ if $p \geq n^{-1 / 2}(\ln n)^{2}$, and $W$ is a suitably large constant. where we have an $O(\ln \ln n p / \ln q)$ term instead of $W$. This improves the previously known lower bound with an $O(\ln \ln n p / \ln q)$ term instead of $W$. We also obtain a slight improvement on the upper bound, using an upper bound on the number of acyclic orientations of an undirected graph. We also analyze a polynomial-time heuristic to find a large induced dag and show that it produces a solution whose size is at least $\log _{q} n p+\Theta\left(\sqrt{\log _{q} n p}\right)$.

Keywords: Random Graphs, Directed Graphs, Concentration, Largest induced acyclic subgraph, Martingales

\section{Introduction}

By a simple directed graph, we mean a directed graph having no 2-cycles. Throughout the paper, we assume, w.l.o.g., that $V=\{1,2, \ldots, n\}$. Given a directed graph $D=(V, A)$, we want to find the maximum size (i.e. number of vertices) of an induced dag in $D$, denoted by mas $(D)$. We study this parameter both theoretically and algorithmically for random digraphs. We obtain improved lower and upper bounds on mas $(D)$ and the results are described in Sections 2 and 3 . Some algorithmic aspects are studied in Section 4

We study the following model of a simple random digraph introduced in [16]. In what follows, $p \leq 0.5$ is a real number. Throughout the paper, we use $q$ to denote $(1-p)^{-1}$ and $w$ to denote $n p$. 
Model $\mathcal{D}(n, p)$ : Let the vertex set be $V=\{1,2, \ldots, n\}$. Choose each undirected edge joining distinct elements of $V$ independently with probability $2 p$. For each chosen $\{u, v\}$, independently orient it in one of the two directions $\{u \rightarrow v, v \rightarrow u\}$ in $D$ with equal probability $=1 / 2$. The resulting directed graph is an orientation of a simple graph, i.e., there are no 2-cycles.

Throughout this paper, we observe the following convention: for a sequence of events $\left\{E=E_{n}\right\}$ defined over $\{D \in \mathcal{D}(n, p) \| p=p(n)\}_{n=0}^{\infty}$, " $E$ holds with high probability" or "asymptotically almost surely $E$ holds" both mean that $\operatorname{Pr}\left[E_{n}\right] \rightarrow 1$ as $n \rightarrow \infty$.

The theoretical aspects of this problem were studied initially by Subramanian [16] and later Spencer and Subramanian [15] obtained the following result:

Theorem 1.1 [15] Let $D \in \mathcal{D}(n, p)$ and $w=n p$. There is a sufficiently large constant $C$ such that : If $p$ satisfies $w \geq C$, then a.a.s,

$$
\operatorname{mas}(D) \in\left[\left(\frac{2}{\ln q}\right)(\ln w-\ln \ln w-O(1)),\left(\frac{2}{\ln q}\right)(\ln w+3 e)\right]
$$

where $q=(1-p)^{-1}$.

Thus, $\operatorname{mas}(D)$ is concentrated in an integer band of width $O\left(\frac{\ln \ln w}{\ln q}\right)$. For $p \leq n^{-0.5}$, this width can become quite large, for example, for $p=D / n$, it is $\Theta(n)$. We initially establish an "essentially" $\sqrt{n}$ width for all ranges of $p$ by using a version (see [1]) of Azuma's Inequality.

Theorem 1.2 Let $D \in \mathcal{D}(n, p), 0<p \leq 1 / 2$. Then, for any $\omega=\omega(n)$ such that $\omega \rightarrow \infty$ as $n \rightarrow \infty$, with high probability,

$$
|\operatorname{mas}(D)-E[\operatorname{mas}(D)]| \leq \omega \sqrt{n}
$$

Theorem 1.2 provides a sharper concentration than 1.1 when $p=o\left(n^{-1 / 2} \ln \ln n\right)$. For larger values of $p$, however, Theorem 1.1 provides a better concentration gap. For such values of $p$, we improve this further by obtaining the following lower bound on $\operatorname{mas}(D)$.

Theorem 1.3 Let $D \in \mathcal{D}(n, p)$. There is a large positive constant $W$ such that : If $n^{-1 / 2}(\ln n)^{2} \leq p \leq$ $1 / 2$, then almost surely,

$$
\operatorname{mas}(D) \geq\left(\frac{1}{\ln q}\right)(2 \ln n p-W)
$$

Thus the width of concentration is reduced to $O(1 / \ln q)$, which is $O(1 / p)$. The concentration gap of Theorem 1.1 is therefore improved for all ranges of $p$ except for the small range $c n^{-1 / 2} \ln \ln n \leq p \leq$ $c^{\prime} n^{-1 / 2}(\ln n)^{2}\left(c, c^{\prime}\right.$ are constants). 
The gap between $2 \log _{q} n p$ and the lower bound in Theorem 1.3 is $O(1 / \ln q)=O\left(p^{-1}\right)$, which can become large when $p$ is small. This gap can be reduced to an absolute constant when $p$ is slightly larger:

Theorem 1.4 There exists a large positive constant $W$ such that for every small constant $\epsilon>0$, the following is true : Given $D \in \mathcal{D}(n, p)$, if $p$ is such that $n^{-1 / 3+\epsilon} \leq p \leq 1 / 2$, then a.a.s

$$
\operatorname{mas}(D) \geq \frac{2 \ln n p}{\ln q}-W
$$

The proofs of Theorems 1.2 1.3 and 1.4 are presented in Section 2 The proof of Theorem 1.2 uses a martingale-based inequality, whereas those of Theorems 1.3 and 1.4 are based on the Second Moment Method.

In Section 3 , using upper bounds on the number of acyclic orientations of an undirected graph, we obtain the following slight improvement (not asymptotic) on the upper bound of Theorem 1.1 .

Theorem 1.5 Let $D \in \mathcal{D}(n, p)$. If p satisfies $n^{-1 / 2+\epsilon} \leq p \leq 0.5$ where $\epsilon>0$ is any constant, then a.a.s

$$
\operatorname{mas}(D) \leq\left\lceil\frac{2}{\ln q}(\ln n p+\ln (7 e)\rceil+1\right.
$$

This method also indicates why we are unlikely to get any asymptotic improvement in the upper bound, at least using the first moment method. Alongwith the argument used in the previous upper bound, it indicates the possibility of a new proof to concentrate the number of acyclic orientations of a random graph $G_{n, p}$, denoted by $a o(G)$.

\subsection{The algorithmic aspects}

By $\operatorname{MAS}(D, k)$, we denote the following computational problem : Given a simple directed graph $D=$ $(V, A)$ and $k$, determine if $\operatorname{mas}(D) \geq k$. A related problem is the Feedback Vertex Set $F V S(D, k)$ problem, in which a set of $k$ vertices have to be removed to make the remaining (di)graph acyclic. $\operatorname{MAS}(D, k)$ is the complement of the $\operatorname{FVS}(D, k)$ (fvs) problem, since on removing a feedback vertex set, the remaining vertices induce an acyclic subgraph in $D$. Both the fvs and mas problems come up in various applications in computer science, such as in proving partial correctness of programs [4], in deadlock recovery in operating systems [12], and in VLSI design [14]. They have been widely studied (see [3], [11] for a survey of related problems). Solving $\operatorname{MAS}(D, k)$ for arbitrary digraphs would allow us to solve both of these problems.

However, $\operatorname{MAS}(D, k)$ is known to be NP-complete [5]. In fact, even the approximation version is known to be hard [8] when the input is an arbitrary digraph: for some $\epsilon>0$, a polynomial-time approximation algorithm with an approximation ratio of $O\left(n^{\epsilon}\right)$ is not possible unless $P=N P$.

If we focus on random digraphs drawn from $\mathcal{D}(n, p)$, it was shown in [16] that a greedily built solution is of size at least $\epsilon\left(\log _{q} n p\right)$ (for $p=\Omega\left(n^{-1}\right)$ ), for every fixed $\epsilon<1$. It was also predicted in [16] that $\epsilon$ can be made to approach 1 asymptotically. In Section 4 , we improve this algorithmic result further by studying a heuristic which combines greedy and brute-force approaches as follows. We first apply the greedy heuristic to get a partial solution whose size is nearly $\log _{q} n p-c \sqrt{\log _{q} n p}$ for some arbitrary constant $c$. Then, in the subgraph induced by those vertices each of which can be safely added to the partial solution, we find an optimal solution by brute-force and combine it with the partial solution. In 
Section 4, it is shown that (for every fixed $p$ ) this modified approach produces a solution whose size is at least $\log _{q} n p+c \sqrt{\log _{q} n p}$. This results in an additive improvement of $\Theta\left(\sqrt{\log _{q} n p}\right)$ over the simple greedy approach. The improvement is mainly due to the fact we stop using the greedy heuristic at a point where it is possible to apply brute-force efficiently. This approach is similar to (and was motivated by) the "expose-and-merge" approach used in [7] for finding large independent sets in $\mathcal{G}(n, 1 / 2)$.

\section{Improved lower bounds for $\operatorname{mas}(D(n, p))$}

First, we prove Theorem 1.2 We use the following theorem on the concentration of functions satisfying Lipschitz condition.

Theorem 2.1 Let $f(D)$ be any real-valued function defined over all directed graphs on $V=[n]$ satisfying $\left|f(D)-f\left(D^{\prime}\right)\right| \leq 1$ whenever $D$ and $D^{\prime}$ differ only on the edges incident at a single vertex. Then, for $D \in \mathcal{D}(n, p)$, and any $t>0$,

$$
\operatorname{Pr}[|f(D)-E[f(D)]| \geq t] \leq 2 e^{-t^{2} / 2 n}
$$

Proof: : The proof arguments are based on constructing a vertex-exposure martingale and applying Azuma's inequality to it. They are essentially the same as those employed in [13] and also follows as a consequence of Theorem 7.4.2 of [1].

Lemma 2.2 For any two digraphs $D, D^{\prime}$ which differ only in edges incident at a single vertex,

$$
\left|\operatorname{mas}(D)-\operatorname{mas}\left(D^{\prime}\right)\right| \leq 1
$$

Proof: : Let $v$ be the vertex at whose incident edges $D$ and $D^{\prime}$ differ. Remove the vertex $v$ from $D$ and $D^{\prime}$. The resulting digraphs are now identical, and so $\operatorname{mas}(D \backslash v)=\operatorname{mas}\left(D^{\prime} \backslash v\right)$. Now, restoring the vertex $v$ to $D$ and $D^{\prime}$ can only retain or increase the size of the optimal DAGs in $D$ and $D^{\prime}$. So either both $\operatorname{mas}(D)$ and $\operatorname{mas}\left(D^{\prime}\right)$ remain the same or both rise by 1 , or only one of them rises. In all cases, we have $\left|\operatorname{mas}(D)-\operatorname{mas}\left(D^{\prime}\right)\right| \leq 1$.

Proof: (of Theorem 1.2): The proof follows from Theorem 2.1 and Lemma 2.2 Let $Y=\operatorname{mas}(D)$ be the random variable denoting the size of the largest induced acyclic subgraph. Then, $Y$ satisfies the requirements of Theorem 2.1 and hence applying Theorem 2.1, we have

$$
\operatorname{Pr}[|Y-E[Y]| \geq t] \leq 2 e^{-t^{2} / 2 n} .
$$

Taking $t=\omega \sqrt{n}$, where $\omega$ is any asymptotically increasing function of $n$, the result follows.

Proofs of new lower bounds : We now present the proofs of Theorems 1.3 and 1.4 Before that, we introduce some facts, notations and definitions: (F1) For $p, 0 \leq p \leq 0.5$, it is easy to verify that $p \leq \ln q$ and $\ln q \leq(1.5) p$. (N1) We use the standard notation $(n)_{b}$ to denote the expression $n(n-1) \ldots(n-b+1)$ defined for all positive integers $n$ and $b$. (D1) Given a directed graph $D=(V, E)$, a topological ordering 
of a set $A \subseteq V$ is a permutation $\sigma:[|A|] \rightarrow A$ such that every arc in $D[A]$ is of the form $\sigma(i) \rightarrow \sigma(j)$, where $i<j$. (D2) A pair of vertices $\sigma(i), \sigma(j) \in A$ is said to be consistent with the topological ordering $\sigma$ if they do not induce a backward arc in $D[A]$, i.e. an arc of the form $\sigma(i) \rightarrow \sigma(j)$, where $i>j$. (D3) Let $S_{n}$ denote the set of all permutations of $[n]$. Given a permutation $\sigma \in S_{n}$, an inversion is defined to an (unordered) pair of elements $i, j \in[n]$, such that $i<j$ but $\sigma(i)>\sigma(j)$. We use the following identity from [17].

Lemma 2.3 [17] For $\sigma \in S_{n}$, let $i(\sigma)$ denote the number of inversions in $\sigma$. Then

$$
\sum_{\sigma \in S_{n}} q^{i(\sigma)}=(1+q)\left(1+q+q^{2}\right) \ldots\left(1+q+q^{2}+\ldots+q^{n-1}\right)
$$

Proofs (of Theorems 1.3 and 1.4) The proofs of both theorems are essentially the same and we give a common proof highlighting at appropriate places where they differ. Given $D \in \mathcal{D}(n, p)$, consider the random variable

$$
Y=Y(b)=\mid\{(A, \sigma): A \subseteq V,|A|=b, \sigma \text { is a permutation of } A\} \mid .
$$

Let $T_{i}=\left(A_{i}, \sigma_{i}\right), A_{i} \subseteq V,\left|A_{i}\right|=b$ be the $i$-th ordered $b$-set. Define an indicator random variable $Y_{i}$ which is set to 1 if $\sigma_{i}$ is a topological ordering for $D\left[A_{i}\right]$, and zero otherwise. Then,

$$
Y=Y(b)=\sum_{i=1}^{(n)_{b}} Y_{i} ; \text { Also, for each } i, \quad E\left[Y_{i}\right]=\operatorname{Pr}\left[Y_{i}=1\right]=(1-p)^{\left(\begin{array}{c}
b \\
2
\end{array}\right)}
$$

Hence, by linearity of expectation, $E[Y]=\sum_{i=1}^{(n)_{b}} E\left[Y_{i}\right]=(n)_{b}(1-p)^{\left(\begin{array}{c}b \\ 2\end{array}\right)}$. Define

$$
b^{*}=\left\lfloor\frac{2 \ln n p}{\ln q}-X\right\rfloor=\frac{2 \ln n p}{\ln q}-X-\delta
$$

where $(i) X=W$ if $p \geq n^{-1 / 3+\epsilon}$ and $X=W /(\ln q)$ if $p \geq n^{-1 / 2}(\ln n)^{2}$ and $(i i) \delta, 0 \leq \delta<1$, is defined to be the fractional part of the expression $2 \log _{q} n p-X$. We first prove the first moment at $b^{*}$ goes to infinity as $n \rightarrow \infty$.

Lemma 2.4 At $b=b^{*}, E[Y] \rightarrow \infty$ as $n \rightarrow \infty$.

Proof : The proof is by substituting the value of $b^{*}$ in $E\left[Y\left(b^{*}\right)\right]$ :

$$
E[Y]=(n)_{b}(1-p)^{\left(\begin{array}{l}
b \\
2
\end{array}\right)} \geq(n-b)^{b}(1-p)^{\left(\begin{array}{l}
b \\
2
\end{array}\right)}=n^{b}(1-b / n)^{b}(1-p)^{\left(\begin{array}{l}
b \\
2
\end{array}\right)}
$$

But for $p \geq n^{-1 / 2}(\ln n)^{2}, b / n \leq \frac{2(\ln n p)}{n(\ln q)} \leq \frac{2(\ln n p)}{n p}$ and hence $b^{2} / n \leq \frac{4(\ln n p)^{2}}{n p^{2}}=o(1)$. Hence, $b^{2} / n \rightarrow 0$ as $n \rightarrow \infty$, so that $(1-b / n)^{b} \rightarrow 1$ as $n \rightarrow \infty$. Hence, as $n \rightarrow \infty$,

$E[Y] \approx n^{b}(1-p)^{\left(\begin{array}{c}b \\ 2\end{array}\right)}=\left(n(1-p)^{(b-1) / 2}\right)^{b} \geq\left(n(1-p)^{\ln n p / \ln q}\right)^{b}=(n / n p)^{b}=p^{-b} \geq p^{-\ln n p / \ln q}$

Since $p \geq n^{-1 / 2}(\ln n)^{2}, n p=\omega(1)$, whereas $\ln q \leq \ln 2$. Therefore, $\ln n p / \ln q=\omega(1)$ and so, $E[Y] \rightarrow \infty$ as $n \rightarrow \infty$. 
Now we continue with the proof of the main theorems. It is based on the Second Moment method. By applying Chebyshev's Inequality to the random variable $Y$, we have

$$
\operatorname{Pr}[Y=0] \leq \operatorname{Var}(X) / E[Y]^{2}=\left(E\left[Y^{2}\right]-E[Y]^{2}\right) / E[Y]^{2}
$$

But $Y=\sum_{i} Y_{i}$ is the sum of indicator variables and hence by standard arguments [[1]],

$$
\operatorname{Var}(Y)=\sum_{i} \operatorname{Var}\left(Y_{i}\right)+\sum_{i \neq j} \operatorname{Cov}\left(Y_{i}, Y_{j}\right) \leq E[Y]+\sum_{i \neq j} \operatorname{Cov}\left(Y_{i}, Y_{j}\right)
$$

where the sum is over ordered pairs $(i, j)$ and $\operatorname{Cov}\left(Y_{i}, Y_{j}\right)=E\left[Y_{i} Y_{j}\right]-E\left[Y_{i}\right] E\left[Y_{j}\right]$ denotes the covariance of the random variables $Y_{i}$ and $Y_{j}$. Clearly, if $\left|A_{i} \cap A_{j}\right|<2$, then $Y_{i}$ and $Y_{j}$ are independent and hence $\operatorname{Cov}\left(Y_{i}, Y_{j}\right)=0$. On the other hand, even if $\left|A_{i} \cap A_{j}\right|=b, Y_{i}$ and $Y_{j}$ could still be different random variables having non-zero covariance, since the permutations $\sigma_{i}$ and $\sigma_{j}$ could differ. Hence, only the pairs $(i, j)$ for which $2 \leq\left|A_{i} \cap A_{j}\right| \leq b$ are of interest. Now,

$$
\begin{aligned}
\sum_{i \neq j} \operatorname{Cov}\left(Y_{i}, Y_{j}\right) & \leq \sum_{i \neq j} E\left[Y_{i} Y_{j}\right]=\sum_{i \neq j} E\left[Y_{i}\right] \cdot E\left[Y_{j} \mid Y_{i}=1\right] \\
& =\sum_{i} E\left[Y_{i}\right] \sum_{j: 2 \leq\left|A_{i} \cap A_{j}\right| \leq b} E\left[Y_{j} \mid Y_{i}=1\right] \\
& \leq \sum_{i} E\left[Y_{i}\right] E[Y] \cdot M=E[Y]^{2} \cdot M
\end{aligned}
$$

where $M$ denotes $\max _{i} \sum_{j: 2 \leq\left|A_{i} \cap A_{j}\right| \leq b} E\left[Y_{j} \mid Y_{i}=1\right] / E[Y]$.

If it can be shown that $M=o(1)$, this implies that $\operatorname{Var}(Y) \leq E[Y]+o\left(E[Y]^{2}\right)$ and hence that $\operatorname{Pr}(Y=0) \leq(E[Y])^{-1}+o(1)=o(1)$ since $E[Y] \rightarrow \infty$. This establishes that with high probability, $Y=Y\left(b^{*}\right)>0$ and hence $\operatorname{mas}(D) \geq b^{*}$. Hence, it suffices to only show that $M=o(1)$.

For $T_{i}, T_{j}, 1 \leq i, j \leq(n)_{b}$, define $A_{i, j}:=A_{i} \cap A_{j}$, and let $\left|A_{i, j}\right|=l, 2 \leq l \leq b$. Without loss of generality, assume that $A_{j}=\{1,2, \ldots b\}, A_{i, j}=\{1,2, \ldots l\}$. We shall be concerned with the ordering of the vertices in the sets $A_{i}$ and $A_{j}$ restricted to $A_{i, j}$, so let the permutation $\sigma_{i}$ restricted to $A_{i, j}$ be the identity, and the $\sigma_{j}$ be $\sigma$ (actually $\sigma_{j}$ relative to $\sigma_{i}$, that is $\sigma=\sigma_{j} \sigma_{i}^{-1}$ ).

$$
\begin{aligned}
E\left[Y_{j} \mid Y_{i}=1\right] & =\operatorname{Pr}\left[Y_{j}=1 \mid Y_{i}=1\right] \\
& =\prod_{1 \leq r<s \leq b} \operatorname{Pr}\left[(\sigma(s), \sigma(r)) \notin D\left[A_{j}\right] \mid Y_{i}=1\right] \\
& =\left(\prod_{1 \leq r<s \leq b ; s>l}(1-p)\right) \cdot\left(\prod_{1 \leq r<s \leq l} \operatorname{Pr}\left[(\sigma(s), \sigma(r)) \notin D\left[A_{j}\right] \mid Y_{i}=1\right]\right)
\end{aligned}
$$

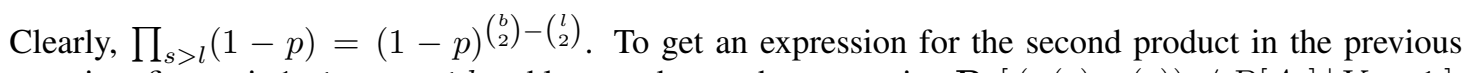
equation, fix a pair $1 \leq r<s \leq l$ and let $p_{r, s}$ denote the expression $\operatorname{Pr}\left[(\sigma(s), \sigma(r)) \notin D\left[A_{j}\right] \mid Y_{i}=1\right]$. Since $\left.\sigma_{i}\right|_{A_{i, j}}$ is the identity permutation over $A_{i, j}$ and since $Y_{i}=1$, it follows that $(s, r) \notin D\left[A_{i, j}\right]$. This 
means that if $\sigma(r)>\sigma(s)$, then $\sigma(r), \sigma(s)$ form an inversion in $\sigma$ and we want $(r, s) \notin D\left[A_{i, j}\right]$. Hence, $p_{r, s}=\operatorname{Pr}\left[(\sigma(r), \sigma(s)) \notin D\left[A_{i, j}\right] \mid(r, s) \notin D\left[A_{i, j}\right]\right]=(1-2 p) /(1-p)$. Otherwise, $(r, s)$ do $n o t$ form an inversion pair and $p_{r, s}=1$. Hence the second product evaluates to exactly $\left(\frac{1-2 p}{1-p}\right)^{i(\sigma)}$.

As a result, we have

$$
E\left[Y_{j} \mid Y_{i}=1\right]=\operatorname{Pr}\left[Y_{j}=1 \mid Y_{i}=1\right]=(1-p)^{\left(\begin{array}{c}
b \\
2
\end{array}\right)-\left(\begin{array}{l}
l \\
2
\end{array}\right)}\left(\frac{1-2 p}{1-p}\right)^{i(\sigma)}
$$

$M$ is therefore:

$$
\begin{aligned}
M & =\max _{i} \sum_{2 \leq l \leq b} \sum_{j:\left|A_{i} \cap A_{j}\right|=l} \operatorname{Pr}\left[Y_{j}=1 \mid Y_{i}=1\right] / E[Y] \\
& =\max _{i}\left(\sum_{2 \leq l \leq b} \sum_{j:\left|A_{i} \cap A_{j}\right|=l}(1-p)^{-\left(\begin{array}{c}
l \\
2
\end{array}\right)} \sum_{\pi \in S_{b} / S_{l}} \sum_{\sigma \in S_{l}}\left(\frac{1-2 p}{1-p}\right)^{i(\sigma)}\right) \cdot(n)_{b}^{-1}
\end{aligned}
$$

where $S_{k}$ is the group of all permutations of a $k$-element set. Once $A_{i}$ is fixed, $A_{j}$ such that $\left|A_{i} \cap A_{j}\right|=$ $l$, can be chosen in $\left(\begin{array}{c}n-b \\ b-l\end{array}\right)\left(\begin{array}{l}b \\ l\end{array}\right)$ ways. Given $A_{i}, A_{j}$ and a permutation $\sigma$ over $A_{i, j}$, a permutation $\pi$ over $A_{j}$ (whose restriction to $A_{i} \cap A_{j}$ is $\sigma$ ) can be chosen in $b ! / l$ ! ways. Thus,

$$
\begin{aligned}
& M=\sum_{l=2}^{b}(n)_{b}^{-1}\left(\begin{array}{l}
b \\
l
\end{array}\right)\left(\begin{array}{c}
n-b \\
b-l
\end{array}\right)(b ! / l !) \sum_{\sigma \in S_{l}}\left(\frac{1-2 p}{1-p}\right)^{i(\sigma)}(1-p)^{-\left(\begin{array}{c}
l \\
2
\end{array}\right)} \\
& =\sum_{l=2}^{b}\left(\begin{array}{l}
n \\
b
\end{array}\right)^{-1}\left(\begin{array}{l}
b \\
l
\end{array}\right)\left(\begin{array}{c}
n-b \\
b-l
\end{array}\right)\left((1-p)^{\left.-\left(\begin{array}{l}
l \\
2
\end{array}\right) / l !\right)} \sum_{\sigma \in S_{l}}\left(\frac{1-2 p}{1-p}\right)^{i(\sigma)}\right. \\
& =\sum_{l=2}^{b}\left(\begin{array}{l}
b \\
l
\end{array}\right)^{2} \frac{(n-b)_{b-l}}{(n)_{b}}(1-p)^{-\left(\begin{array}{l}
l \\
2
\end{array}\right)} \sum_{\sigma \in S_{l}}\left(\frac{1-2 p}{1-p}\right)^{i(\sigma)} \\
& \leq \sum_{l=2}^{b}\left(\begin{array}{l}
b \\
l
\end{array}\right)^{2} \frac{(1-l / n)^{-l}}{n^{l}}(1-p)^{-\left(\begin{array}{c}
l \\
2
\end{array}\right)} \sum_{\sigma \in S_{l}}\left(\frac{1-2 p}{1-p}\right)^{i(\sigma)}
\end{aligned}
$$

Since $l \leq b$, we have $(1-l / n)^{-l} \leq(1-b / n)^{-b} \rightarrow 1$ as $n \rightarrow \infty$. Again, with $r=(1-2 p) /(1-p)=$ $1-p /(1-p)<1$ and applying Lemma 2.3 .

$$
\begin{aligned}
M & \leq \sum_{l=2}^{b}\left(\begin{array}{l}
b \\
l
\end{array}\right)^{2} n^{-l}(1-p)^{-\left(\begin{array}{c}
l \\
2
\end{array}\right)} \prod_{i=1}^{l}\left(\frac{1-r^{i}}{1-r}\right) \\
& =\sum_{l=2}^{b}(1-r)^{-l}\left(\begin{array}{l}
b \\
l
\end{array}\right)^{2} n^{-l}(1-p)^{-\left(\begin{array}{l}
l \\
2
\end{array}\right)} \prod_{i=1}^{l}\left(1-r^{i}\right)=\sum_{l=2}^{b} A_{l}^{l}
\end{aligned}
$$

We bound the value of $A_{l}^{l}$ in the following two ways:

$$
A_{l}^{l} \leq p^{2}\left(\begin{array}{l}
b \\
l
\end{array}\right)^{2}\left(\frac{1}{n p(1-p)^{l / 2}}\right)^{l}
$$


by substituting the value of $r$ in terms of $p$ and simplifying, and,

$$
A_{l}^{l} \leq\left(\begin{array}{l}
b \\
l
\end{array}\right)^{2} l !\left(\frac{1}{n(1-p)^{l / 2}}\right)^{l}, \text { since } r<1 .
$$

Case I : $2 \leq l \leq t$, where $t=2 / \ln q$ :

$$
A_{l}^{l} \leq\left(\begin{array}{l}
b \\
l
\end{array}\right)^{2} l !\left(\frac{1}{n(1-p)^{l / 2}}\right)^{l} \leq\left(\frac{b^{2}}{n(1-p)^{l / 2}}\right)^{l} \leq\left(\frac{b^{2}}{n(1-p)^{t / 2}}\right)^{l} \leq\left(\frac{b^{2} e}{n}\right)^{l}=o(1)
$$

since $b^{2} e / n \leq 1 /(\log n)^{2}=o(1)$.

Therefore, $\sum_{l=2}^{t} A_{l}^{l} \leq \sum_{l=2}^{\infty}\left(b^{2} e / n\right)^{l}=s^{2} /(1-s) \leq s^{2}(1+2 s)=o(1)$ where $s=b^{2} e / n=o(1)$.

Case II: $t<l \leq b / 2$ :

$$
\begin{aligned}
A_{l}^{l} & \leq\left(\begin{array}{c}
b \\
l
\end{array}\right)^{2} l !\left(\frac{1}{n(1-p)^{l / 2}}\right)^{l} \leq\left(\frac{e b^{2}}{n l(1-p)^{l / 2}}\right)^{l} \leq\left(\frac{e b^{2}}{n t(1-p)^{l / 2}}\right)^{l} \\
& \leq\left(\frac{e b(\ln n p)}{n(1-p)^{b / 4}}\right)^{l} \leq\left(\frac{e b(\ln n p) \sqrt{n p}}{n}\right)^{l} \leq\left(\frac{e b \sqrt{p}(\ln n p)}{\sqrt{n}}\right)^{l} .
\end{aligned}
$$

Now $b \sqrt{p}(\ln n p)=O\left(p^{-1 / 2}(\ln n)^{2}\right)=O\left(n^{1 / 4}(\ln n)\right)$ because of our assumption about $p$. Hence, the summation can be upper-bounded by $(b / 2)\left(\frac{e b \sqrt{p}(\ln n p)}{\sqrt{n}}\right)^{t}$, which is less than $(b / 2)\left(n^{-1 / 8}\right)^{t}$, and is clearly $o(1)$.

Until now the proof arguments for both Theorem 1.3 and 1.4 are the same. The proofs vary for the remaining cases. First, we complete the

Proof of Theorem 1.3: Our assumption is $p \geq n^{-1 / 2}(\ln n)^{2}$.

Case III $: b / 2<l \leq b:$

$$
\begin{aligned}
A_{l}^{l} / p^{2} & \leq\left(\begin{array}{l}
b \\
l
\end{array}\right)^{2}\left(\frac{1}{n p(1-p)^{l / 2}}\right)^{l} \leq\left(\frac{e^{2} b^{2}}{n p l^{2}(1-p)^{l / 2}}\right)^{l} \leq\left(\frac{4 e^{2}}{n p(1-p)^{b / 2}}\right)^{l} \\
& \leq\left(\frac{4 e^{2}(1-p)^{\delta / 2}}{n p(1 / n p)(1-p)^{-X / 2)}}\right)^{l} \leq\left(\frac{4 e^{2}}{e^{W / 2}}\right)^{l}
\end{aligned}
$$

for $W \geq 8$. The summation can therefore be upper-bounded by $(b / 2)\left(4 / e^{2}\right)^{-b / 2}$, which is again $o(1)$, as $\lim _{n \rightarrow \infty} b=\infty$. This establishes that $M=o(1)$. So, we conclude that the probability that there is no topologically ordered set of size $b=\left\lfloor 2 \log _{q} n p-W /(\ln q)\right\rfloor$, goes to zero for a suitably chosen constant $W$ (in fact $W=8$ suffices). This proves the lower bound and hence completes the proof of Theorem 1.3

Proof of Theorem 1.4: The proof is along the same lines and the only difference occurs when $l>b / 2$. Case III (in the previous proof) gets split into two subcases : 
Case IIIa : $b / 2<l \leq b-c^{\prime}$, where $c^{\prime}=8 / \ln q$ :

$$
\begin{aligned}
A_{l}^{l} / p^{2} & \leq\left(\begin{array}{l}
b \\
l
\end{array}\right)^{2}\left(\frac{1}{n p(1-p)^{l / 2}}\right)^{l} \leq\left(\frac{e^{2} b^{2}}{n p l^{2}(1-p)^{l / 2}}\right)^{l} \leq\left(\frac{4 e^{2}}{n p(1-p)^{\left(b-c^{\prime}\right) / 2}}\right)^{l} \\
& \leq\left(\frac{4 e^{2}(1-p)^{(X+\delta) / 2}}{n p(1 / n p)(1-p)^{-c^{\prime} / 2}}\right)^{l} \leq\left(\frac{4 e^{2}}{e^{4}}\right)^{l}
\end{aligned}
$$

Again the summation is upper bounded by $(b / 2)\left(4 / e^{2}\right)^{-b / 2}$, which is $o(1)$.

Case IIIb: $b-c^{\prime}<l \leq b$ : Here we look at the ratio of successive terms:

$$
\begin{aligned}
A_{l+1}^{l+1} / A_{l}^{l} & =\left(\frac{b-l}{l+1}\right)^{2}\left(\frac{1}{n(1-p)^{l}}\right)\left(\frac{1-r^{l+1}}{1-r}\right) \\
& \geq \frac{1}{2 b^{2}}\left(\frac{(1-p)^{1+X+\delta}}{n p(1 / n p)^{2}(1-p)^{-c^{\prime}}}\right) \geq\left(\frac{n p(1-p)^{c^{\prime}+1+X+\delta}}{2 b^{2}}\right)=\Omega\left(n p^{3}(\ln n p)^{-2}\right)
\end{aligned}
$$

For $p \geq n^{-1 / 3+\epsilon}, n p^{3}(\ln n p)^{-2}=\omega(1)$. Hence, the ratio $A_{l+1}^{l+1} / A_{l}^{l} \geq 1$ in the stated range of $l$. So the function $A_{l}^{l}$ is increasing in the range $b-c^{\prime} \leq l \leq b$, and the maximum value is therefore attained at $l=b$. For this value of $l$ (using the assumption that $W$ is a constant) :

$$
\begin{aligned}
A_{b}^{b} & \leq\left(\begin{array}{l}
b \\
b
\end{array}\right)^{2}\left(\frac{1}{n p(1-p)^{b / 2}}\right)^{b}=\left((1-p)^{(W+\delta) / 2}\right)^{b}=O\left((1-p)^{W(\ln n p) /(\ln q)}\right) \\
& =O\left(e^{-W(\ln n p)}\right)=O\left((n p)^{-W}\right)=o\left(n^{-2}\right)
\end{aligned}
$$

Therefore, the summation is upper-bounded as follows:

$$
\sum_{b-c^{\prime}}^{b} A_{l}^{l} \leq c^{\prime} A_{b}^{b}=o\left(p^{-1} n^{-2}\right)=o(1)
$$

This establishes that $M=o(1)$ and we conclude that the probability that there is no topologically ordered set of size $b=\left\lfloor 2 \log _{q} n p-W\right\rfloor$, goes to zero for a suitably chosen constant $W$. This proves the lower bound and hence completes the proof of Theorem 1.4 .

\section{Upper Bound}

In this section, we prove Theorem 1.5 . Throughout this section, we define $b$ to be $b=\left\lceil\frac{2(\ln w+\ln (7 e))}{(\ln q)+1}\right\rceil$. Consider an undirected graph $G=(V, E)$. Let $a o(G)$ denote the number of acyclic orientations of $G$. An orientation $O$ of $G$ is obtained by directing each edge $\{i, j\} \in E(G)$ from $i$ to $j$ (indicated by $(i, j)$ ) or vice-versa to get the digraph $D=(V, A), A=O(G)$. An orientation $O$ is acyclic if the resulting digraph $D$ has no directed cycles. 
Let $a(m)$ be the maximum number of acyclic orientations of any simple undirected graph $Y$, where $Y$ has $b$ vertices and $m$ edges. Then the probability that the induced digraph on a fixed b-set $Y$ of $V[D]$, $D \in \mathcal{D}(n, p)$ is a dag is upper-bounded by:

$$
\begin{aligned}
\operatorname{Pr}[D[Y] \text { is acyclic }] & =\mathbf{E}_{X \in \mathcal{G}(b, 2 p)} \frac{a o(X)}{2^{|E(X)|}} \\
& \leq \sum_{m=0}^{\left(\begin{array}{c}
b \\
2
\end{array}\right)} \frac{a(m)}{2^{m}}\left(\begin{array}{c}
\left(\begin{array}{c}
b \\
2
\end{array}\right) \\
m
\end{array}\right)(2 p)^{m}(1-2 p)^{\left(\begin{array}{c}
b \\
2
\end{array}\right)-m}
\end{aligned}
$$

We use the simple upper bound $a(m) \leq(d+1)^{b}$, where $d=2 m / b$ is the average degree of any such graph $Y$, obtained in [9]. Let $Z$ denote the number of edges in the random subgraph of $\mathcal{G}(n, 2 p)$ induced by $Y$. Since $G[Y]$ is drawn according to the distribution $\mathcal{G}(b, 2 p), Z$ has expectation given by $\mathbf{E}[Z]=\left(\begin{array}{l}b \\ 2\end{array}\right) 2 p$.

The variable $Z$ is in fact binomial and using Chernoff-Hoeffding large-deviation bounds [[10], [1]], the probability of its being much larger than the expected value can be tightly bounded:

$$
\begin{aligned}
\operatorname{Pr}[Z>3 \mathbf{E}[Z]] & \leq\left(e^{2} 3^{-3}\right)^{\mathbf{E}[Z]}<e^{-2 b(p / \ln q) \ln n p}<(n p)^{-2(p / \ln q) b} \\
& \leq n^{-2 b(p / \ln q)(1 / 2+\epsilon)}=n^{-b(p / \ln q)(1+2 \epsilon)}
\end{aligned}
$$

For $0 \leq p \leq 1 / 2,0.7 \leq p / \ln q \leq 1$ [[16]]. Now, the sum in (8) can be broken into two parts, as $m \leq 3 \mathbf{E}[Z]$ and $m>3 \mathbf{E}[Z]$ :

$$
\begin{aligned}
& \operatorname{Pr}[D[Y] \text { is acyclic }] \leq \sum_{m \leq\lfloor 3 \mathbf{E}[Z]\rfloor} a(m)\left(\begin{array}{c}
b \\
2 \\
m
\end{array}\right)(p)^{m}(1-2 p)^{\left(\begin{array}{c}
b \\
2
\end{array}\right)-m} \\
& +\sum_{m>\lfloor 3 \mathbf{E}[Z]\rfloor+1} \frac{a(m)}{2^{m}}\left(\begin{array}{c}
\left(\begin{array}{c}
b \\
2
\end{array}\right) \\
m
\end{array}\right)(2 p)^{m}(1-2 p)^{\left(\begin{array}{c}
b \\
2
\end{array}\right)-m} \\
& \leq a(\lfloor 3 \mathbf{E}[Z]\rfloor)(1-p)^{\left(\begin{array}{c}
b \\
2
\end{array}\right)}+\left(\frac{b !}{2^{3 \mathbf{E}[Z]}}\right) \operatorname{Pr}[Z>3 \mathbf{E}[Z]] \\
& \leq(6 b p+1)^{b}(1-p)^{\left(\begin{array}{l}
b \\
2
\end{array}\right)}+\frac{b !}{2^{3 \mathbf{E}[Z]}} n^{-b(0.7)(1+2 \epsilon)} \\
& \leq(6 b p+1)^{b}(1-p)^{\left(\begin{array}{l}
b \\
2
\end{array}\right)}+(b !) n^{-b[(0.7)(1+2 \epsilon)+(6 \ln 2)(\ln n p / \ln n)(p / \ln q)]}
\end{aligned}
$$

Since $(\ln n p / \ln n) \geq(1 / 2+\epsilon)$ and $(p / \ln q) \geq 0.7$, on applying the union bound over all $b$-sized subsets of $V$, we see that the probability that there exists an acyclic $b$-set $A \subset V$ is at most $\left(\begin{array}{l}n \\ b\end{array}\right)[(6 b p+$ $\left.1)^{b}(1-p)^{\left(\begin{array}{l}b \\ 2\end{array}\right)}+(b !) n^{-b((0.7)(1+2 \epsilon)+0.7}\right]$, Let $P_{b}$ denote $\operatorname{Pr}[\exists Y,|Y|=b, D[Y]$ is acyclic $]$.

$$
\begin{aligned}
P_{b} & \leq\left(\begin{array}{l}
n \\
b
\end{array}\right)\left[(6 b p+1)^{b}(1-p)^{\left(\begin{array}{c}
b \\
2
\end{array}\right)}+(b !) n^{-b(1+2 \epsilon)}\right] \leq\left(\begin{array}{c}
n \\
b
\end{array}\right)(6 b p+1)^{b}(1-p)^{\left(\begin{array}{c}
b \\
2
\end{array}\right)}+n^{-2 b \epsilon} \\
& =\left(\begin{array}{l}
n \\
b
\end{array}\right)(6 b p+1)^{b}(1-p)^{\left(\begin{array}{c}
b \\
2
\end{array}\right)}+o(1) \leq\left((6 e n p+e n / b)(1-p)^{(b-1) / 2}\right)^{b}+o(1)
\end{aligned}
$$




$$
\begin{aligned}
& \leq\left((6 e n p+e n(\ln q) /(2 \ln w))(1-p)^{(b-1) / 2}\right)^{b}+o(1) \\
& \leq\left(((6+o(1)) e n p)(1-p)^{(b-1) / 2}\right)^{b}+o(1)
\end{aligned}
$$

The last expression in the previous inequality goes to zero since the base (which is raised to its $b$-th power) is less than 1 for our definition of $b$. As a result, we get that $\operatorname{mas}(D) \leq 2\left\lceil\log _{q} w+\log _{q}(7 e)\right\rceil+1$. We observe that this expression is marginally better than the expression obtained in [15], although the asymptotics are the same, i.e. $\operatorname{mas}(D)=O\left(p^{-1} \ln n p+p^{-1}\right)$.

\section{An efficient heuristic with improved guarantee}

It was shown in [16] that, for every fixed $\epsilon<1$, with probability $1-o(1)$, every maximal induced dag is of size is at least $\epsilon\left(\log _{q} n p\right)$. A maximal solution can be obtained in polynomial time. It was also mentioned in [16] that one can possibly set $\epsilon=1$. We further refine this analysis and show that the above statement holds for some $\epsilon=\epsilon(n) \rightarrow 1$. Precisely, we have the following strengthening of Theorem 3.1 of [16].

Theorem 4.1 Let $p=p(n) \leq 0.5$ be such that $w=n p \geq X$ for some sufficiently large constant $X>0$. Then, for $D \in \mathcal{D}(n, p)$, with probability $1-o(1)$, every maximal induced dag is of size at least $\delta\left(\log _{q} w\right)$ where $\delta=1-\frac{2(\ln \ln w)+10}{\ln w}$.

The proof of this theorem is skipped in this abstract and follows by refining the analysis given in [16].

We present below another efficient heuristic which will be analyzed and shown to have an additive improvement (for every fixed $p \leq 0.5)$ of $\Theta\left(\sqrt{\log _{q} w}\right)$ over the guarantee given in [16] and in Theorem 4.1. It is similar to a heuristic presented in [7] for finding large independent sets in $G \in \mathcal{G}(n, 1 / 2)$. We show that, for every fixed $c>0$, one can find in polynomial time an induced DAG of size at least $\left\lfloor\log _{q} w+c \sqrt{\log _{q} w}\right\rfloor$.

Let $C$ be the set of those vertices which could be each individually added to the greedy solution. The idea is to construct greedily a solution $A$ of size $g(n, p, c)=\left\lceil\log _{q} w-c \sqrt{\log _{q} w}\right\rceil$ and then add an optimal solution (found by an exhaustive search) in the subgraph induced by vertices in $C$. We will show that exhaustive search can be done in polynomial time and yields (almost surely) a solution of size $2 c \sqrt{\log _{q} w}$. As a result, we finally get a solution of the stated size. The algorithm is described below.

$\operatorname{MAXDAG}(D=(V, E), c)$

1. Choose and fix a linear ordering $\sigma$ of $V$.

2. $c^{\prime}:=1.2 c ; A:=\emptyset ; B:=V$.

3. while $B>n / 2$ and $|A|<g\left(n / 2, p, c^{\prime}\right)$ do

4. Let $u$ be the $\sigma$-smallest vertex in $B$.

5. If $D[A \cup\{u\}]$ induces an acyclic subgraph then add $u$ to $A$.

6. remove $u$ from $B$. endwhile

7. if $|A|<g\left(n / 2, p, c^{\prime}\right)$, then Return FAIL and halt. 
8. $C:=\{u \in B:(u, v) \notin E, \forall v \in A\} ; \quad \mu=|B|(1-p)^{|A|}$.

9. if $|C| \notin[(0.9) \mu,(1.1) \mu]$ then Return FAIL.

10. for each $X \subset C:|X|=\left\lceil 2 c^{\prime} \sqrt{\log _{q} w / 2}+2 \log _{q} 0.9-W\right\rceil$ do

11. if $D[X]$ is acyclic then Return $D[A \cup X]$ and halt. endfor

12. Return FAIL.

We analyze the above algorithm and obtain the following result.

Theorem 4.2 Let $p=p(n) \leq 0.5$ be such that $p \geq \tau$ for some fixed but arbitrary positive constant $\tau$ and let $D \in \mathcal{D}(n, p)$. Then, for every constant $c \geq 1$, with probability $1-o(1), \operatorname{MAXDAG}(D, c)$ will output an induced acyclic subgraph of size at least $b^{\prime}=\left\lfloor\left(1+\epsilon^{\prime}\right) \log _{q} n p\right\rfloor$, where $\epsilon^{\prime}=c / \sqrt{\log _{q} n p}$.

Proof: : Without loss of generality, we assume that $c$ is sufficiently large.

Correctness : First, we prove the correctness. Note that $D[A]$ is always an induced acyclic subgraph. Also, each $u \in C$ is such that $D[A \cup\{u\}]$ is an acyclic subgraph with $u$ as a sink vertex (having zero out-degree). Hence, any acyclic subgraph $D[X]$ present as a subgraph in $D[C]$ can be safely added to $A$ so that $D[A \cup X]$ also induces an acyclic subgraph of $D$.

Time Complexity : It is easy to see that the running time is polynomial except for the for loop of lines 10 and 11. The maximum number of iterations of the for loop is at most

$$
\begin{aligned}
\left(\begin{array}{c}
(1.1) \cdot|B|(1-p)^{|A|} \\
\left\lfloor c^{\prime} \sqrt{\log _{q} w / 2}\right\rfloor
\end{array}\right) & \leq\left(\begin{array}{c}
(2.2) \cdot p^{-1} q^{c^{\prime} \sqrt{\log _{q} w / 2}} \\
\left\lfloor c^{\prime} \sqrt{\log _{q} w / 2}\right\rfloor
\end{array}\right) \\
& \leq q^{2 c^{\prime 2}\left(\log _{q} w / 2\right)} \cdot\left((2.2) p^{-1}\right)^{2 c^{\prime} \sqrt{\log _{q} w / 2}}=O\left(n^{O(1)}\right)
\end{aligned}
$$

since $p$ is a constant. Since each iteration takes polynomial time, the algorithm always finishes in polynomial time.

Analysis : Consider the following events defined as

$$
\begin{array}{ll}
\mathcal{E}_{1}: & |A|<g\left(n / 2, p, c^{\prime}\right) ; \\
\mathcal{E}_{2}: & |C| \notin[(0.9) \mu,(1.1) \mu] \\
\mathcal{E}_{3}: & \operatorname{mas}(D[C])<\left\lceil 2 c^{\prime} \sqrt{\log _{q} w / 2}+2 \log _{q} 0.9-W\right\rceil ;
\end{array}
$$

If none of these events holds, then the algorithm will succeed and output a solution whose size is

$$
\begin{aligned}
|A \cup X| & \geq \log _{q}(w / 2)-c^{\prime} \sqrt{\log _{q}(w / 2)}+2 c^{\prime} \sqrt{\log _{q} w / 2}+2 \log _{q} 0.9-W \\
& \geq\left(1+\epsilon^{\prime}\right)\left(\log _{q} w\right)+\left(c^{\prime}-c\right) \sqrt{\log _{q} w / 2}+2 \log _{q} 0.9-W-\log _{q} 2 \\
& \geq\left(1+\epsilon^{\prime}\right)\left(\log _{q} w\right)+(0.2 c) \sqrt{\log _{q} w / 2}+2 \log _{q} 0.9-\left(W+\log _{q} 2\right) \\
& \geq\left(1+\epsilon^{\prime}\right)\left(\log _{q} w\right)
\end{aligned}
$$


We have

$$
\operatorname{Pr}\left(\overline{\mathcal{E}_{1}} \overline{\mathcal{E}_{2}} \overline{\mathcal{E}_{3}}\right)=\operatorname{Pr}\left(\overline{\mathcal{E}_{1}}\right) \cdot \operatorname{Pr}\left(\overline{\mathcal{E}_{2}} \mid \overline{\mathcal{E}_{1}}\right) \cdot \operatorname{Pr}\left(\overline{\mathcal{E}_{3}} \mid \overline{\mathcal{E}_{1}} \overline{\mathcal{E}_{2}}\right) \geq 1-\sum_{i \leq 3} \operatorname{Pr}\left(\mathcal{E}_{i} \mid \wedge_{j<i} \overline{\mathcal{E}_{j}}\right)
$$

Let $V_{1}$ denote the set of first $n / 2$ vertices of $\sigma$. Now using Theorem 4.1, the greedy algorithm run on the first $n / 2$ vertices yields with probability $1-o(1)$, an acyclic subgraph of size

$\delta\left(\log _{q}(w / 2)\right) \geq \log _{q} w / 2-2\left(\log _{q}(\ln w / 2)\right)-(10 / \ln q) \geq g\left(n / 2, p, c^{\prime}\right)=\left\lceil\log _{q} w / 2-c^{\prime} \sqrt{\log _{q} w / 2}\right\rceil$,

with probability $1-o(1)$. Here, $\delta$ is defined in Theorem 4.1. Hence, $\operatorname{Pr}\left(\mathcal{E}_{1}\right)=o(1)$.

For any fixed vertex $u \in B$,

$$
\operatorname{Pr}(u \in C)=\operatorname{Pr}(\forall v \in A,(u, v) \notin E)=(1-p)^{|A|} .
$$

Hence

$$
\mu=E[|C|]=|B| \cdot(1-p)^{|A|} .
$$

Since $|C|$ is the sum of $|B|$ identical and independent indicator random variables, by applying ChernoffHoeffding bounds (see [10, 1]), we get that

$$
\operatorname{Pr}(|C| \notin[(0.9) \mu,(1.1) \mu]) \leq 2 e^{-\mu / 300} .
$$

Since $|A|=g\left(n / 2, p, c^{\prime}\right)$, we deduce that

$$
\mu \approx|B| \cdot 2 q^{c^{\prime}} \sqrt{\log _{q} w / 2} / w,
$$

after justifiably ignoring the effect of the ceiling function used in the definition of $g\left(n / 2, p, c^{\prime}\right)$. Given that $\overline{\mathcal{E}_{1}}$ holds and also since $|B| \geq n / 2$, it is easy to verify that $\mu \rightarrow \infty$ as $n \rightarrow \infty$. Hence $\operatorname{Pr}\left(\mathcal{E}_{2} \mid \overline{\mathcal{E}_{1}}\right)=o(1)$.

Given that neither of $\mathcal{E}_{1}$ and $\mathcal{E}_{2}$ holds, it follows that $|C| \geq(0.9) \mu \approx(0.9) \cdot p^{-1} \cdot q^{c^{\prime}} \sqrt{\log _{q} w / 2}$. Hence, using $q \leq 2$ and applying Theorem 1.4 .

$$
\operatorname{mas}(D[C]) \geq\left\lfloor 2 c^{\prime} \sqrt{\log _{q} w / 2}\right\rfloor+2 \log _{q} 0.9-W \geq\left\lfloor 2 c^{\prime} \sqrt{\log _{q} w / 2}\right\rfloor-1
$$

with probability $1-o(1)$. This establishes that $\operatorname{Pr}\left(\mathcal{E}_{3} \mid \overline{\mathcal{E}_{1}} \overline{\mathcal{E}_{2}}\right)=o(1)$. It then follows from 9 that $\operatorname{MAXDAG}(D, c)$ outputs a solution of required size with probability $1-o(1)$.

\section{Remarks on approximating $\operatorname{MAS}(D, k)$ and minimum fvs}

The MAXDAG $(D, c)$ algorithm in the previous section gives an average-case approximation ratio of 2 for the $\operatorname{MAS}(D, k)$ problem. Since the vertices left on removing an induced acyclic subgraph form a feedback vertex set, we notice that a concentration for the minimum feedback vertex set in random digraphs is also obtained. This follows from Theorems 1.3 As stated in Section 1 a polynomial-time approximation algorithm with a worst-case approximation ratio of $O\left(n^{\epsilon}\right)$ for some fixed $\epsilon$ is NP-hard. However, it can be shown that the algorithm $\operatorname{MAXDAG}(D, c)$ yields on average an approximate solution with an approximation ratio of $\left(1+O\left(\frac{\ln w}{w}\right)\right)$. 


\section{Conclusions}

We studied the problem of determining the mas $(D)$, the size of the largest induced acyclic subgraph in a random directed graph $D=(V, E)$. The range of the concentration of mas $(D)$ is reduced from the previously known $O\left(p^{-1} \ln \ln n p\right)$ to $O(\sqrt{n})$ for all ranges of the arc probability $p=p(n)$, using a martingale-based approach. Using the Second Moment method, the lower bound is improved from $\frac{2}{\ln q}(\ln n p-\ln \ln n p-O(1))$ to $\frac{2}{\ln q}(\ln n p-O(1))$ for all $p \geq n^{-1 / 2}(\log n)^{2}$, and in particular, to

$\frac{2 \ln n p}{\ln q}-O(1)$, for $p \geq n^{-1 / 3+\epsilon}$ for any constant $\epsilon$. Thus, for all $p$, the concentration band is improved to $O\left(\min \left\{p^{-1}, \sqrt{n}\right\}\right)$.

Further, we use an upper bound on the maximum number $a o(G)$ of acyclic orientations of an undirected graph $G=(V, E)$, to get an upper bound on $\operatorname{mas}(D)$. This bound seems to be the best possible, using the first moment method. This is suggested by the result of Kahale and Schulman, who in [6] show that a tight upper bound of the number of acyclic orientations is given by the determinant of the Kirchoff matrix which is asymptotically close (in the exponent) to the simple bound used in Section 3 .

Lastly, we analyse a polynomial time heuristic $\operatorname{MAXDAG}(D, c)$ for getting a large induced acyclic subgraph in a random digraph, and show that for fixed values of the arc-probability $p$, it gives an acyclic subgraph of size at least $\log _{q} w+c \sqrt{\log _{q} w}$ for any constant $c$, which is a slight improvement over the bound of the greedy heuristic MaximalAcyclic $(D)$ given in [16]. In proving this, we also showed that the MaximalAcyclic $(D)$ algorithm itself can be shown to yield a (slightly) larger subgraph than that of Theorem 3.1 of [16] where it was proved only to yield a subgraph of size at least $\epsilon\left(\log _{q} w\right)$, for fixed (but arbitrary) $\epsilon<1$. We improved this by allowing $\epsilon \rightarrow 1$ as $n \rightarrow \infty$.

\section{References}

[1] Alon, N. and Spencer, J.H., (2001). The Probabilistic Method, Wiley International.

[2] Bollobás, B. (2001). Random Graphs (2nd Edition) Camb. Univ. Press.

[3] Cai M., Deng X., and Zang W., (2001). “An Approximation Algorithm for Feedback Vertex Set in Tournaments” SIAM Journal of Computing, 30, 6, 1993-2007.

[4] Floyd, R.W., (1967). “Assigning meaning to programs” (1967) Proc. Symp. Appl. Math. 19 19-32.

[5] M.R. Garey and D.S. Johnson, (1978). Computers and Intractability: A Guide To The Theory of NP-Completeness, W.H.Freeman, San Francisco.

[6] Kahale, N., and Schulman, L.J., (1996). "Bounds on the Chromatic Polynomial and on the number of Acyclic Orientations of a graph", Combinatorica, 16 (3) 383-397.

[7] Krivelevich, M. and Sudakov, B. (1998). "Coloring random graphs", Information Processing Letters, 67, 71-74.

[8] Lund, C. and Yannakakis, M. (1993). "The Approximation of Maximum Subgraph Problems", Proceedings of the 20th International Colloquium on Automata, Languages and Programming (ICALP'93), LNCS 700 40-51. 
[9] Manber, U. and Tompa M. (1984). "The Effect of Number of Hamiltonian Paths on the Complexity of a Vertex-Coloring Problem, SIAM J. Comp., 13, 109-115.

[10] Motwani, R. and Raghavan P., (1995). Randomized Algorithms, Cambridge University Press.

[11] Raman V. and Saurabh S., (2006). "Parameterized algorithms for feedback set problems and their duals in tournaments", Theoretical Computer Science 351 (3) 446-458.

[12] Rosen B. K., (1982). "Robust linear algorithms for cutsets”, J. Algorithms 205-217 3.

[13] Shamir, Eli and Spencer, Joel (1987). "Sharp concentration of the chromatic number on random graphs $G_{n, p}$ ", Combinatorica, 7, 121-129.

[14] Speckenmeyer, E. (1989). "On feedback problems in digraphs", Proceedings of 15th International Workshop on Graph Theoretic Concepts in Computer Science (WG'89), Springer-Verlag, LNCS 411, 218-231.

[15] Spencer, J. and Subramanian, C.R., (2008). "On the size of induced acyclic subgraphs in random digraphs", Disc. Math. and Theoret. Comp. Sci., 10:2, 47-54.

[16] Subramanian, C.R., (2003). "Finding induced acyclic subgraphs in random digraphs", The Electronic Journal of Combinatorics, 10, \#R46.

[17] Stanley, R.P. (1997). Enumerative Combinatorics, Cambridge University Press. 
